\title{
Spectroscopic Studies of Comets 9P/Tempel 1, 37P/Forbes and C/2004 Q2 (Machholz)
}

\author{
Enos Picazzio $^{1}$, Klim I. Churyumov ${ }^{2}$, Larissa S. Chubko ${ }^{3}$, \\ Igor V. Lukyanyk ${ }^{2}$, Valery V. Kleshchonok ${ }^{2}$, \\ Amaury A. de Almeida ${ }^{1}$, and Roberto D. D. Costa ${ }^{1}$ \\ ${ }^{1}$ Department of Astronomy, Institute of Astronomy, Geophysics and Atmospheric Sciences, \\ University of São Paulo, \\ Rua do Matão 1226, Cidade Universitária 05508-900 São Paulo SP, BRAZIL \\ email:picazzio@astro.iag.usp.br \\ ${ }^{2}$ Astronomical Observatory, Kyiv Shevchenko National University, \\ Box 04053, Observatorna str., 3, Kyiv, Ukraine \\ email: klim.churyumov@observ.univ.kiev.ua \\ ${ }^{3}$ National Aviation University, \\ Box 03680, Kosmonavta Komarova ave. 1, Kiev, Ukraine
}

\begin{abstract}
The results of the analysis of the spectra of comets 9P/Tempel 1, 37P/Forbes and C/2004 Q2 (Machholz) observed in 2004-2005 at Observatório do Pico dos Dias (Brazil), and at Mount Pastukhov (SAO, Russia) are presented.
\end{abstract}

Keywords. comets: general, comets: individual (9P/Tempel 1, 37P/Forbes, C/2004 Q2 (Machholz))

\section{Observations and processing of cometary spectra}

High and middle-resolution optical spectra of comets obtained with long-slit spectroscopy allow to (1) calculate some physical parameters of the cometary neutral atmosphere (escape velocities of the gas in the coma, lifetime of particles, etc.) (2) search for new cometary emission lines, (3) estimate parameters of gas and dust activity of the comet nucleus, (4) detect the cometary luminescence continuum of non-solar nature (Churyumov et al. 1994; Churyumov et al. 1999; Lukyanyk \& Churyumov, 2002; Churyumov et al., 2002; Lukyanyk et al., 2002; Picazzio et al., 2002; Picazzio et al., 2006; Chubko et al., 2009).

The spectra of comets $9 \mathrm{P} /$ Tempel 1 and $37 \mathrm{P} /$ Forbes were observed at Observatório do Pico dos Dias (LNA - Laboratório Nacional de Astrofísica), Brasópolis (Brazil) during 3-5 July 2005 with the Cassegrain spectrograph using a 900/500 grating, attached to the Perkin \& Elmer 1.6-m telescope of LNA. In addition spectra of comet 9P/Tempel 1 and C/2004 Q2 (Machholz) were obtained with SCORPIO (Spectra Camera with Optical Reducer for Photometrical and Interferometrical Observations) installed in the prime focus of the 6-m telescope, and with the 1-m Zeiss reflector equipped with the longslit spectrograph of the Special Astrophysical Observatory of the RAS on 14-16 March and 3-4 July 2005 (Mount Pastukhov, Russia). Another 2 spectra of comet C/2004 Q2 (Machholz) were obtained during the night on 17-18 Dec. 2004 also with the 6-m telescope and the MPFS spectrograph of the SAO of RAS. 


\section{Physical parameters of comets 9P/Tempel 1, C/2004 Q2 (Machholz) and 37P/Forbes}

In order to determine some physical parameters of the gaseous components of the neutral cometary atmosphere (the gas component expansion $\mathrm{u}$ and the lifetime of the particles $\tau$ ) we constructed a photometric profile for the $C_{2}, C_{3}$, and $\mathrm{CN}$ emission lines along the slit for comets $9 \mathrm{P} /$ Tempel 1 and 37P/Forbes (Fig. 1). Then the obtained monochromatic profiles were processed by Shul'man's model. Within this model the surface brightness was determined through the following formulas (Shul'man, 1970):

$$
\begin{gathered}
\lg \frac{I(\rho, \varphi+\pi)}{I(\rho, \varphi)}=1.72 \frac{\rho}{r_{0 C}} \sin \Theta_{0} \cos \varphi \\
\frac{1}{2} \lg [I(\rho, \varphi+\pi) I(\rho, \varphi)]=\text { const }+\lg \left[\frac{r_{0 k}}{\rho} \int_{\frac{\rho}{r_{0 k}}}^{\infty} K_{0}(y) d y\right]
\end{gathered}
$$

where $I(\rho, \varphi+\pi)$ and $I(\rho, \varphi)$ represent the brightness surface of emission line along slit, $\rho, \varphi$ are polar coordinates on the picture plane with the polar axis directed to the Sun, $r_{0 c}=\frac{2 u^{2}}{g}$ is the characteristic scale of the spherical symmetry region, $u$ is the expansion velocity, $g$ is the acceleration of molecules in the gravity field of the Sun, $\varphi$ is an angle between the $\mathrm{z}$ axis and g-vector, $K_{0}(y)$ is the modified Bessel function of the second kind.

The physical parameters of the neutral gaseous molecules $C_{2}(5165 \AA), C_{3}(4050 \AA)$, CN (4200 $\AA$ ) (velocity of expansion, lifetime and scale length of parent and daughter molecules) are given in the Table 1 .

From Table 1 we see that the measured expansion velocities of the $C_{2}, C_{3}$ and $\mathrm{CN}$ molecules in the coma of the three comets diverge noticeably from the gas expansion velocity determined by Delsemme's formula

$$
v=\frac{0.58}{\sqrt{r}}
$$

which gives equal velocities for all molecules at the same heliocentric distance.
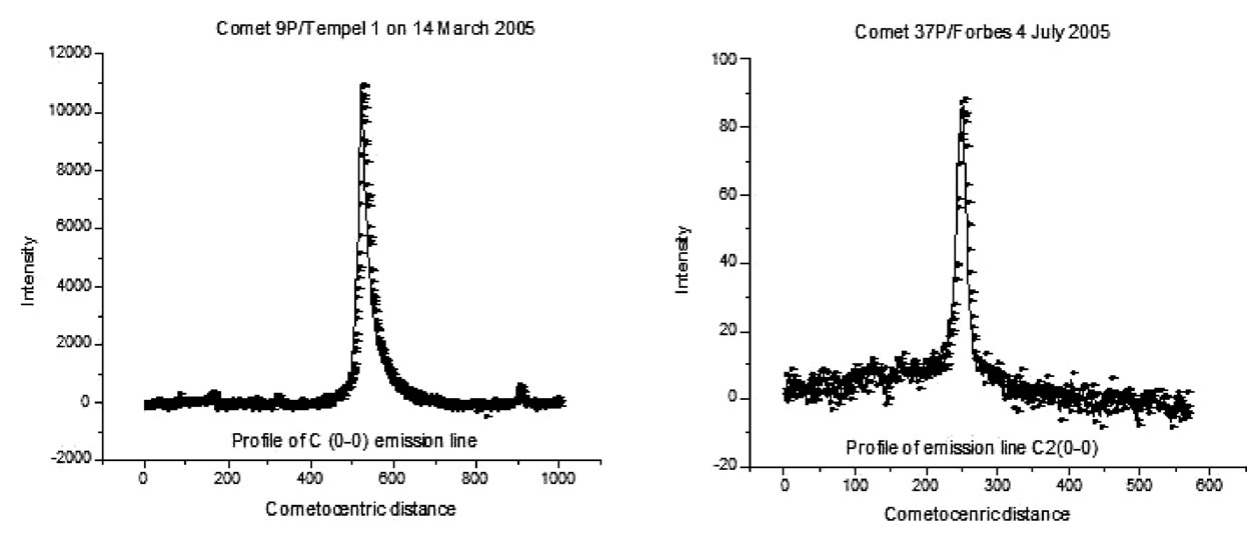

Figure 1. Profiles of brightness in the emission line $C_{2}(0-0)$ in the spectra of comets 9P/Tempel 1 and 37P/Forbes. 
Table 1. Physical parameters of neutral gaseous cometary components of $C_{2}, C_{3}$ and $C N$ (Shul'man's model).

\begin{tabular}{ccccc}
\hline Species & Velocity, $\left[\frac{m}{s}\right]$ & Lifetime, $\left(10^{6} s\right)$ & Comet & Date \\
\hline$C_{2}(5165 \AA)$ & 222 & 1.36 & $9 \mathrm{P} /$ Tempel 1 & $14 / 03 / 2005$ \\
$C_{3}(4050 \AA)$ & 102 & 0.38 & $9 \mathrm{P} /$ Tempel 1 & $14 / 03 / 2005$ \\
$C N(4200 \AA)$ & 67 & 1.35 & $9 \mathrm{P} /$ Tempel 1 & $14 / 03 / 2005$ \\
$C N(4200 \AA)$ & $>300$ & $>50$ & Machholz C $/ 2004 \mathrm{Q} 2$ & $18 / 12 / 2004$ \\
$C_{3}(4050 \AA)$ & 363 & 5.13 & Machholz C $/ 2004 \mathrm{Q} 2$ & $18 / 12 / 2004$ \\
$C_{2}(5165 \AA)$ & 535 & 39.9 & Machholz C $/ 2004 \mathrm{Q} 2$ & $18 / 12 / 2004$ \\
$C_{2}(5165 \AA)$ & 855 & 73.4 & Machholz C/2004 Q2 & $15 / 03 / 2005$ \\
$C_{2}(5165 \AA)$ & 209 & 25.6 & $37 \mathrm{P} /$ Forbes & $04 / 07 / 2005$ \\
\hline
\end{tabular}

\section{Search and detection of cometary luminescence continuum in spectra of comets C/2004 Q2 (Machholz), 9P/Tempel 1 and 37P/Forbes}

A luminescence continuum was detected for the first time in the spectrum of comet 1P/Halley by G. Nazarchuk, who found two broad features with a maximum of intensity near $3950 \stackrel{\AA}{A}$ and $5100 \AA$ (Nazarchuk, 1987; Nazarchuk, 1987). They were part of the scattered solar continuum. Such a phenomenon is connected with the presence of an additional component of a continuous spectrum in cometary radiation. The source of this additional radiation could be the luminescence of organic cometary particles. Hence the spatial distribution of this source should have a very strong concentration close to the comet nucleus. To summarize: a cometary spectrum $I_{\text {com }}$ consists of three components:

$$
I_{\text {com }}(\lambda)=I_{e}(\lambda)+I_{s}(\lambda)+I_{l}(\lambda)
$$

where $I_{e}$ is the cometary emission spectrum, $I_{s}$ is the solar spectrum reflected by cometary dust, $I_{l}$ is the cometary luminescent continuum. Spectral regions with no strong emission lines $\left(I_{e}=0\right)$ are selected for the determination of the level of a luminescent continuum. In these regions of the spectrum it is accepted, that the level of a luminescent continuum does not vary. Thus, for the selected regions of a cometary spectrum it is possible to accept

$$
I_{\text {com }}(\lambda)=k \cdot I_{f}(\lambda)+l
$$

where $I_{\text {com }}(\lambda)$ is the known solar spectrum which is calculated taking into account the spectral resolution of cometary spectrum, its discontinuity, $k$, is the factor which characterizes the reflective ability of the cometary dust, and $l$ is the intensity of the luminescent continuum. In practice the parameters $k$ and $l$ are selected such that the best agreement to a region of the cometary continuum is obtained. The given technique was used for studying a luminescent continuum in the spectra of comets C/2004 Q2 Machholz, 9P/Tempel 1 and $37 \mathrm{P} /$ Forbes (Fig.2).

The maximum of a luminescent continuum for comet C/2004 Q2 Machholz is close to 6300 A (Churyumov, 1999; Lukyanyk \& Churyumov, 2002; Lukyanyk et al., 2002). In this region its intensity reaches $46 \%$ of the total cometary continuum. For comet 9P/Tempel 1 the level of the luminescent cometary continuum is $30 \%$ of the level of the total cometary continuum with the maximum near wavelength $5250 \AA$. For comet $37 \mathrm{P} /$ Forbes the level of the luminescent cometary continuum is $20 \%$ of the level of the total cometary continuum with the maximum near $4500 \AA$. Comparison of the spectra of the three comets shows that new comet C/2004 Q2 (in Oort's sense) has a higher level luminescent continuum, which may indicate a larger number of organic particles. 

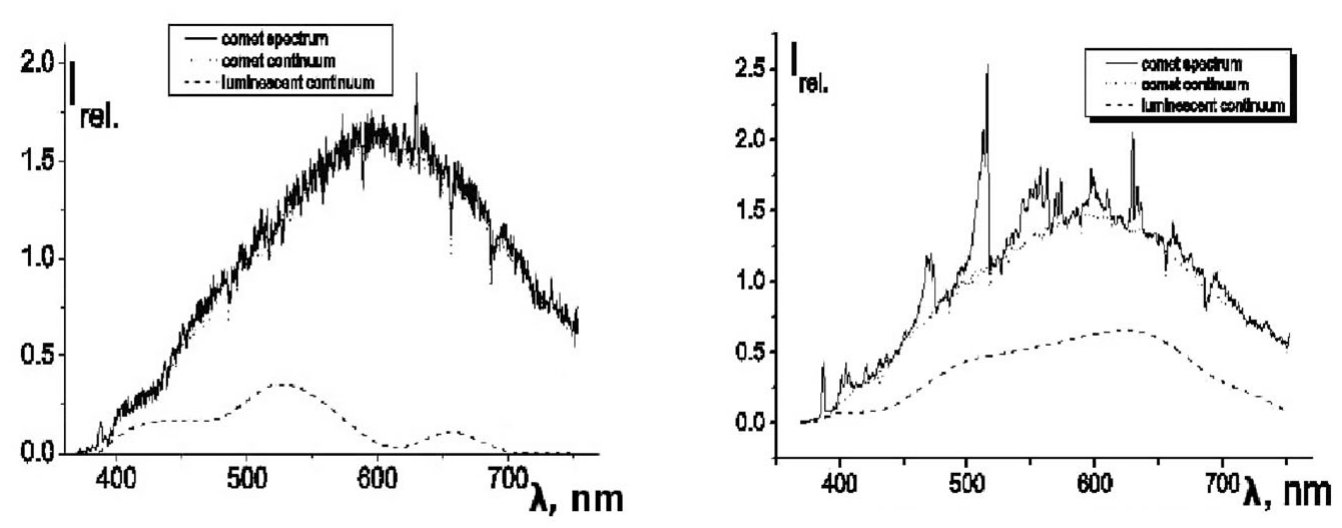

Figure 2. Spectra of comets 9P/Tempel 1 and C/2004 Q2 Machholz with the dedicated cometary continuums and the luminescence cometary dust levels of the total cometary continua.

\section{Conclusion}

The gas component expansion $\mathrm{u}$ and the lifetime of the particles in the comae of comets 9P/Tempel 1, C/2004 Q2 (Machholz) and 37P/Forbes are calculated. The spectra of these comets show evidence for a luminescent cometary continuum which may be connected to the luminescence of organic species in cometary dust particles (e.g. CHON-particles).

\section{References}

Chubko, L. S., Churyumov, K. I., Afanasiev, V. L., Lukyanyk, I. V., \& Kleshchonok, V. V. 2009, Deep Impact as a World Observatory Event: Synergies in Space, Time, and Wavelength, ESO Astrophysics Symposia., p. 197

Churyumov, K. I., Kleshchenok, V. V., \& Vlassyuk, V. V. 1994, Pisma v Astronomicheskij Journal, 20, 9

Churyumov, K. I., Kleshchenok, V. V., \& Mussaev, F. A. 1999, Earth, Moon and Planets, 78, 1

Churyumov, K. I., Lukyanyk, I. V., Afanasiev, V. L. et al. 2002, Proceedings of Asteroids, Comets, Meteors (ACM 2002), p. 657

Lukyanyk, I. V., Churyumov, K. I., Afanasiev, V. L. et al. 2002, Proceedings of Asteroids, Comets, Meteors (ACM 2002), p. 717

Lukyanyk, I. V. \& Churyumov, K. I. 2002, Earth, Moon and Planets, 90, 1

Nazarchuk, H. K. 1987, Kometnyj Tsirkulyar, 372, 2

Nazarchuk, H. K. 1987, Kometnyj Tsirkulyar, 377, 2

Picazzio, E., de Almeida, A. A., Churyumov, K. I., Andrievskii, S. M., \& Lukyanyk, I. V. 2002, Earth, Moon and Planets, 90, 23

Picazzio, E., de Almeida, A. A., Churyumov, K. I., Andrievskii, S. M., Lukyanyk, I. V. 2006, Advances in Space Research, 10, 312

Shulaman, L. M. 1970, Astrometry and Astrophysics, 11, 26 